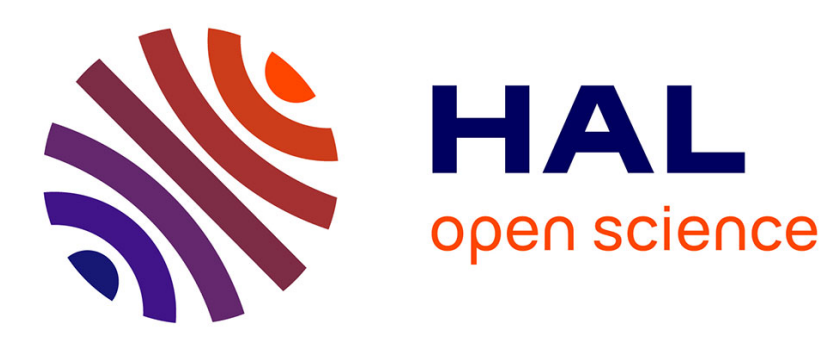

\title{
Pole condensation and the Riemann surface associated with a shock in Burgers' equation
}

\author{
D. Bessis, J.D. Fournier
}

\section{To cite this version:}

D. Bessis, J.D. Fournier. Pole condensation and the Riemann surface associated with a shock in Burgers' equation. Journal de Physique Lettres, 1984, 45 (17), pp.833-841. 10.1051/jphyslet:019840045017083300 . jpa-00232419

\section{HAL Id: jpa-00232419 https://hal.science/jpa-00232419}

Submitted on 1 Jan 1984

HAL is a multi-disciplinary open access archive for the deposit and dissemination of scientific research documents, whether they are published or not. The documents may come from teaching and research institutions in France or abroad, or from public or private research centers.
L'archive ouverte pluridisciplinaire HAL, est destinée au dépôt et à la diffusion de documents scientifiques de niveau recherche, publiés ou non, émanant des établissements d'enseignement et de recherche français ou étrangers, des laboratoires publics ou privés. 
Classification

Physics Abstracts

$03.40 \mathrm{G}-47.40-05.20$

\title{
Pole condensation and the Riemann surface associated with a shock in Burgers' equation
}

\author{
D. Bessis \\ and J. D. Fournier \\ Observatoire, Mont-Gros, BP 139, 06003 Nice Cedex, France
}

Service de Physique Théorique, CEN Saclay, 91191 Gif-sur-Yvette Cedex, France

(Reçu le 15 mai 1984, accepté le 10 juillet 1984)

\begin{abstract}
Résumé. - Pour une condition initiale paradigmatique, nous analysons complètement la structure analytique du champ de vitesses, solution de l'équation de Burgers, en fonction du temps $t$, et de la viscosité $v$. La solution visqueuse a un nombre infini de pôles complexes qui apparaissent soudainement à $t=0_{+}$; quand $v \downarrow 0$, leur condensation sur les lignes de Stokes donne naissance aux singularités inviscides; la surface de Riemann à 3 feuillets associée à ces dernières permet un franchissement analytique du saut du choc.

Abstract. - Using a paradigmatic initial condition, we completely analyse the spatial analytic structure of Burgers velocity field and its dependence on time $t$ and viscosity $v$. The viscous solution has an infinite number of complex poles appearing suddenly at $t=0_{+}$. When $v \downarrow 0$ these poles condense on Stokes lines giving rise to the inviscid singularities; the 3-sheeted Riemann surface associated with the latter provides an analytic path across the jump of the shock.
\end{abstract}

\section{Introduction.}

We are concerned with Burgers' equation

$$
\frac{\partial u}{\partial t}+u \frac{\partial u}{\partial x}=v \frac{\partial^{2} u}{\partial x^{2}}
$$

where $v$, the viscosity, is positive or zero and $u(x, t)$ is the velocity field at point $x$ and time $t$ [1]. For real $x$ and $t$ and a given generic initial condition it is known [2] that the solution exhibits small scale excitations which produce a quasi-shock smoothed by the small finite viscosity (see Fig. 1); in the limit $v \rightarrow 0_{+}$, a preshock (with infinite derivative) and a shock (with dicontinuity) appear (see Fig. 2; Refs. [1-3]). The subject of this paper is to show that these classical results can be understood as the trace in the real domain of phenomena occurring in the complex- $x$ plane $\mathbb{C}$ (some of these were already suggested or partly described in [3-5]). For a paradigmatic initial condition we carry out the complete analysis both in the viscous and inviscid case ; a small- $v$ asymptotics allows us to make the bridge between the two cases. For our example 


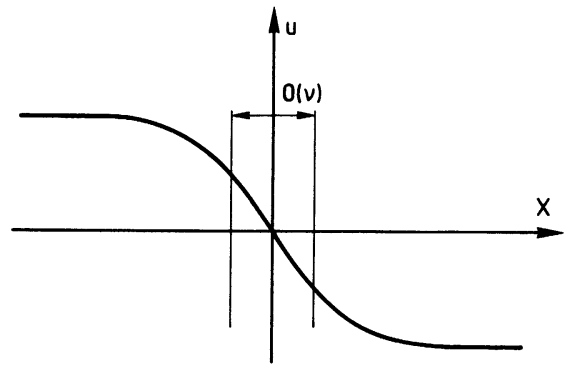

Fig. 1. - Local shape of a quasi-shock which appears for small positive viscosity.

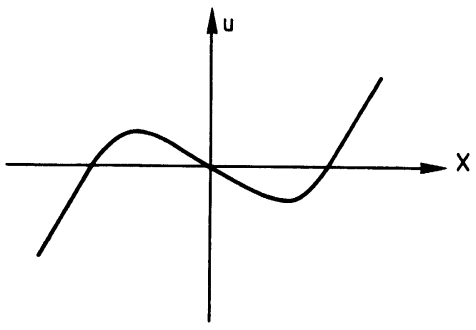

Fig. $2 \mathrm{a}$ - $t$ prior to the preshock time $t_{*}$.

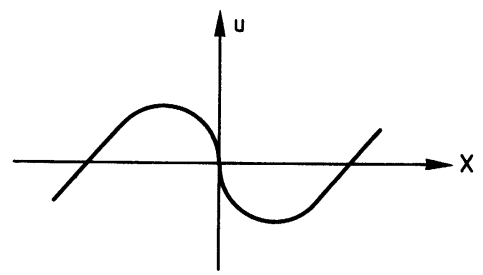

Fig. 2b. $-t=t_{*}$.

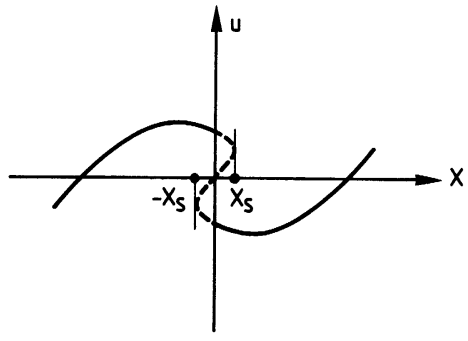

Fig. 2c. $-t$ after $t_{*}$.

Fig. 2. - Local velocity shape in the inviscid case as time increases. 
the solution, which is analytic at $t=0$, nevertheless produces at $t=0_{+}$an infinite number of poles; their dynamics permit us to understand the analytic aspects of the formation of preshock and shock as the viscosity decreases. Previous studies failed to describe these scenarios. In particular we completely clarify the role of the singularities which appear after the preshock time $t_{*}$. These singularities are shown to always be in non-physical Riemann sheets, but they are responsible for the breakdown of various statistical approximations [6].

In this Letter, we shall only summarize and comment on our results, the proofs of which will be published elsewhere.

\section{Paradigmatic initial condition.}

In the inviscid case the velocity has at $t_{*}$ an infinite derivative. This singular behaviour is easily understood when turning to the hydrodynamical interpretation of Burgers' equation : it expresses that the total time derivative of the velocity is zero. Each particle keeping its own initial velocity, fast moving ones catch up to the slow ones (Fig. 2). This accumulation process gives rise at $t_{*}$ to a catastrophy : this takes place at a spatial point which has its origin at time zero in a space region where the difference of velocity between fast and slow particles is maximal with respect to their distance. This region is the neighbourhood of a point $x$ such that

$$
\begin{aligned}
& \left.\frac{\partial u}{\partial x}\right|_{\left(x_{*}, 0\right)}=-M, \quad M>0 ; \\
& \left.\frac{\partial^{2} u}{\partial x^{2}}\right|_{\left(x_{*}, 0\right)}=0 .
\end{aligned}
$$

It is elementary to deduce that

$$
t_{*}=M^{-1}=\left.\left|\inf _{x} \frac{\partial u}{\partial x}\right|_{(x, 0)}\right|^{-1},
$$

and therefore the local generic shape of the initial condition [3] is

$$
u_{0}(x) \equiv u(x, t=0)=-\frac{x}{t_{*}}+b x^{3}+\mathcal{O}\left(x^{4}\right), \quad b>0
$$

Equation (3) is written with the choice $x_{*}=0$ and $u_{0}\left(x_{*}\right)=0$; this is always possible using Galilean invariance.

The initial condition we choose to study has the form

$$
u_{0}(x)=4 x^{3}-\frac{x}{t_{*}}
$$

it is therefore generic for the local study of the shock and preshock; furthermore it allows a complete analytic study.

\section{Inviscid case.}

In the inviscid case, we can write the solution of Burgers' equation for the initial condition (6) as

$$
u(x, t)=\frac{x}{t}+U(x, t),
$$


where $U(x, t)$ satisfies

$$
4 t^{3} U^{3}+\frac{t_{*}-t}{t_{*}}+\frac{x}{t}=0 .
$$

Clearly $U(x, t)$, and therefore $u(x, t)$, is an analytic function of $x$ and appears to be uniform on a three-sheeted Riemann surface. Being representative of the « singular part » of the solution, the time evolution of the topology of this Riemann surface gives a full insight into the dynamics of the system. For fixed positive $t, U(x, t)$ has three singularities : the point at infinity which is a third-order branch point, plus two second-order branch point $x_{\mathrm{s}}$ and $-x_{\mathrm{s}}$ given by

$$
x_{\mathrm{s}}=\frac{i}{3 \sqrt{3}} t_{*}^{-3 / 2} t^{-1 / 2}\left(t_{*}-t\right)^{3 / 2} .
$$

Starting from $t=0$ at infinity, the moving square root singularities $x_{\mathrm{s}}$ and $-x_{\mathrm{s}}$ come down along the imaginary axis [8]; they meet at $t_{*}$ and they move away along the real axis. However this description is much too simple, because the Riemann surface being three-sheeted, these singularities are not present on all sheets. At this point it is necessary to define the physical Riemann sheet, as the extension in the complex plane of the neighbourhood of the physical real domain. For $t<t_{*}$ the physical sheet and the physical region are shown on figure 3 , where the cuts represent the lines of connection towards the other sheets. After $t_{*}$ the shock divides the

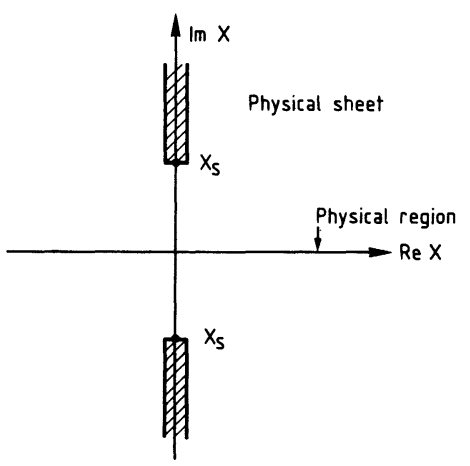

Fig. 3. - The physical Riemann sheet and the corresponding singularities. For $0<t<t_{*}$ in the inviscid case.

physical region into two different domains. The physical sheet is now the union of two separate pieces, the cut of separation being the imaginary axis. The singularities $x_{\mathbf{s}}$ and $-x_{\mathbf{s}}$ are present in neither piece of the physical sheet. These unphysical singularities allow for the existence of a path which connects the left and right sides of the shock (see Fig. 4). Starting from the point R on the right physical region, we first move into the complex right physical sheet, then pass the cut into a second sheet, make a complete turn round $-x_{\mathrm{s}}$ in this second sheet, go into the next sheet, make a complete turn round $+x_{\mathrm{s}}$ and come finally to the left physical region at point $\mathrm{L}$. This path is nothing but the complex extension of the "skeleton path " represented on figure $2 \mathrm{c}$.

A systematic way of describing the situation near time $t_{*}$ is to introduce the topology of the Riemann surface associated with equation (8). Its time dependence is visualized on figures $5 a, b, c$. 


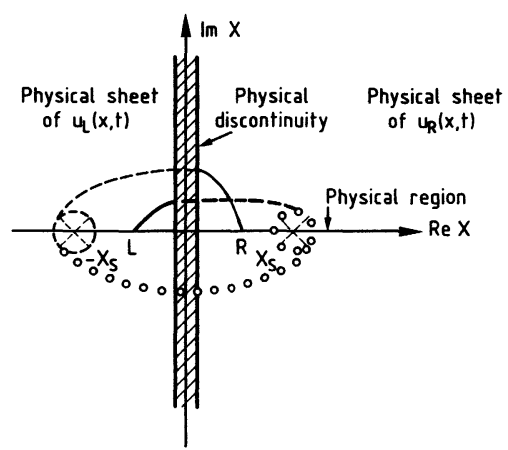

Fig. 4. - The physical Riemann sheets, for $t>t_{*}$ in the inviscid case. The square root singularities $\pm x_{\mathrm{s}}$ are on other sheets. There is a connection between the right physical region and the left one through a path which partly belongs to a third sheet; this path is represented with the following symbols : — physical region; --- left analytic continuation of the right physical region: 000 unphysical sheet; :-- right analytic continuation of the left physical region.

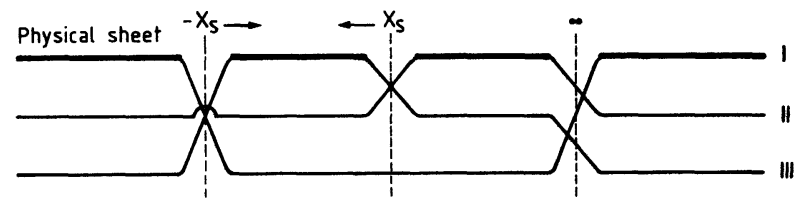

Fig. 5a. $-0<t<t_{*}$. The singularity $x_{\mathrm{s}}$ is not present on sheet III, while $-x_{\mathrm{s}}$ is absent on sheet II ; they are both present on sheet I (physical sheet); the point at infinity is singular on all sheets.

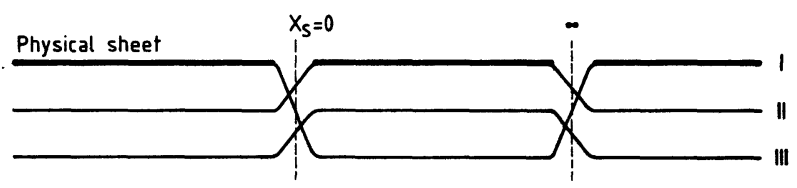

Fig. $5 \mathrm{~b} .-t=t_{*} \cdot x_{\mathrm{s}}$ and $-x_{\mathrm{s}}$ have collided on the real axis giving rise to a branch point of order 3 (preshock); this singularity as well as the one at intmity are present on all sheets.

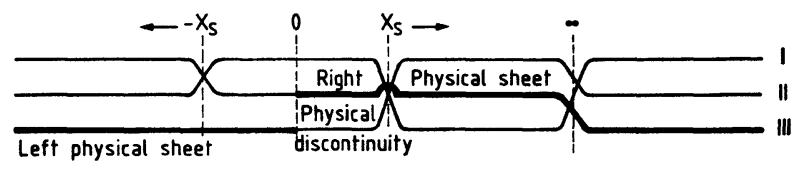

Fig. 5c. $-t>t_{*}$. The singularities $-x_{\mathrm{s}}$ and $x_{\mathrm{s}}$ are now non-physical, the physical sheets are sheets II and III, which are connected locally through sheet I; see the path on figure 4 .

Fig. 5. - Topology of the Riemann surface of the solution in the inviscid case. 


\section{Viscous case.}

We now turn to the finite viscosity case. As is well known [12] Burgers' equation may be mapped into the heat equation [13]. Setting

$$
u(x, t)=-2 v \frac{\partial}{\partial x} \log \theta(t, x),
$$

one gets

$$
\frac{\partial \theta}{\partial t}=v \frac{\partial^{2} \theta}{\partial x^{2}}
$$

the solution of which can be written in our case as

$$
\theta(t, x)=(4 \pi v t)^{-1 / 2} \exp \left[-x^{2} / 4 v t\right] E(x, t)
$$

with

$$
E(x, t)=\int_{-\infty}^{+\infty} \mathrm{d} y \exp \left[-\frac{1}{2 v}\left[y^{4}+\frac{1}{2}\left(\frac{1}{t}-\frac{1}{t_{*}}\right) y^{2}-\frac{1}{t} x y\right]\right] .
$$

It follows from (12) and (13) that $\theta(t, x)$ is an entire function of $x$; therefore the velocity will be a meromorphic function of $x$, the poles of which sit at the zeros of $E$. It can be shown that the order (characterization of the large distance behaviour) of $E(x, t)$ is $4 / 3$ for any strictly positive time; therefore using Hadamard's theorem [18], it follows that, the order being not integer, the number of zeros is necessarily infinite; thus the velocity has an infinite number of poles at any positive time. Using the Hadamard decomposition theorem, and noting that due to the odd parity of $u(x, t)$, poles appear in opposite pairs, one can write $u(x, t)$ as

$$
u_{v}(x, t)=\frac{x}{t}-\sum_{a_{n} \in \mathrm{D}} \frac{4 v x}{x^{2}-a_{n}^{2}(t, v)}-\sum_{a_{n} \in \partial \mathrm{D}} \frac{2 v x}{x^{2}-a_{n}^{2}(t, v)} .
$$

Here $\mathrm{D}$ is the right part of the complex plane, and $a_{n}(t, v)$ the location in $\mathrm{D}$ of the $n$th pole. Formula (14) will be the key of the condensation process of poles; it is also reminiscent of the polar representation of reference [5] in which analogous systems are studied. However, if finite, the number of poles appears to be a constant of motion. In our case, the number of poles being infinite, it allows for the astonishing situation that at $t=0$ there are no poles at all, while there is an infinite spontaneous generation of poles at $t=0_{+}$. This infiniteness will also allow us to get $v i a$ the pole condensation a non-trivial limit when $v \rightarrow 0_{+}$.

\section{From small to zero viscosity.}

Using saddle point methods we study the location and density of this set of infinite poles for small viscosity. The poles are located near the Stokes lines whose shape evolves according to figures $6 \mathrm{a}, \mathrm{b}, \mathrm{c}$.

In the limit $v \rightarrow 0_{+}$, the distance between two nearby poles tends to zero as $v$ and poles condense along the Stokes line. We introduce the density of poles as being

$$
\rho_{v}\left(a_{n}, t\right)=\frac{v}{a_{n+1}(t, v)-a_{n}(t, v)}
$$




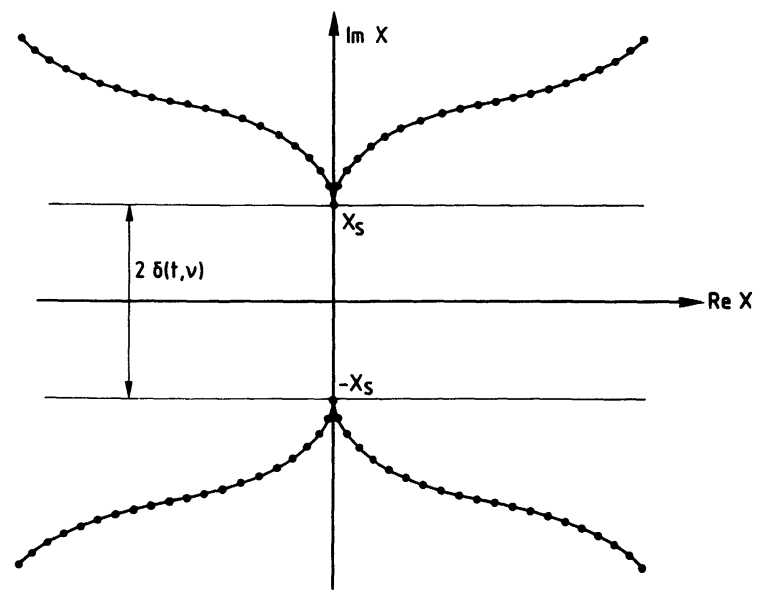

Fig. 6a. $-0<t<t_{*}$. For small $v$ the distance $\delta(t, v) \simeq\left|x_{\mathrm{s}}(t)\right|$ decreases from $\infty$ to $\delta_{\min }=\mathcal{O}(v)$. (See [8-11]).

Fig. $6 \mathrm{~b} .-t=t_{*}$.
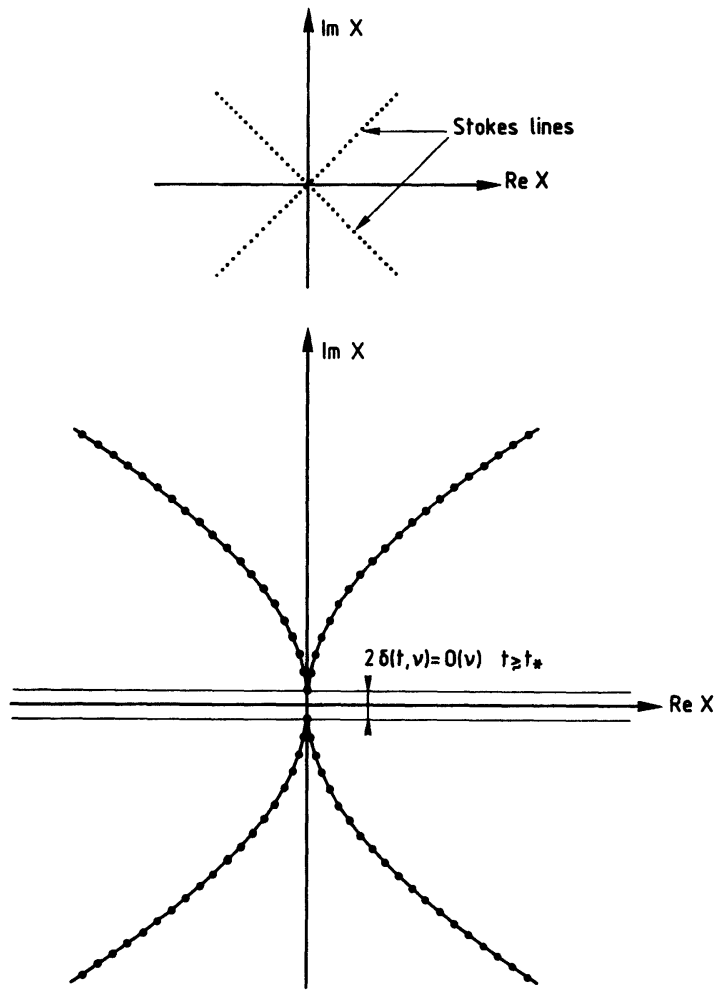

Fig. 6c. $-t>t_{*}$. For $t \gtrsim t_{*}$ and small $v, \delta(t, v)$ is independent of $t$ and is $\mathcal{O}(v)$; eventually it increases when time elapses, see [9]; this corresponds to the erosion of small scale excitation by the viscous dissipation. In the zero viscosity limit, $\delta$ becomes zero; the physical region is separated into two parts (Fig. 4) with a discontinuity (see (21), (22) and [20]).

Fig. 6. - The location of the poles of the velocity in the complex position plane for small viscosity, as time elapses. There is a countable infinity of such poles, all located in the neighbourhood of the represented Stokes lines when the viscosity tends to zero. Their relative distance tends to zero : they " condense ", and the Stokes lines are then frontiers which support a density of poles from which the order of the singularities of the inviscid case may be recovered. 
and its limit

$$
\rho(z, t)=\left.\lim _{\substack{v \rightarrow 0_{+} \\ n \rightarrow \infty}} \rho_{\nu}\left(a_{n}, t\right)\right|_{z=a_{n}} .
$$

Formula (14) in this limit is replaced by an integral representation

$$
u(x, t)=\frac{x}{t}-4 x \int_{(\mathbf{S})} \frac{\rho(z, t)}{x^{2}-z^{2}} \mathrm{~d} z,
$$

where (S) are the Stokes lines situated in the right half of the complex plane. Let us describe two particularly interesting results.

At $t=t_{*}$, the Stokes lines are the lines $z=|z| \mathrm{e}^{ \pm i \pi / 4}$, and the density reads

$$
\rho\left(z, t_{*}\right)=z^{1 / 3} 4^{-4 / 3} 3^{1 / 2} \pi^{-1} \mathrm{e}^{-i \pi / 3} t_{*}^{-4 / 3} ;
$$

when inserted in (17), gives

$$
u\left(x, t_{*}\right)=\frac{x}{t_{*}}\left(\frac{x}{4}\right)^{1 / 3} t_{*}^{-4 / 3} ;
$$

this is precisely the cubic shape of the inviscid preshock which may be obtained directly from equation (8).

The same kind of calculation allows the reconstruction of the inviscid analytic structure before $t_{*}$ (Fig. 6a), namely the correct discontinuity around the square root branch points $\pm x_{\mathrm{s}}$.

For $t>t_{*}$ and small positive viscosity, the Stokes lines (Fig. 6c) remain at a distance of order $v$ from the real axis; they are tangent to the imaginary axis and the local density (neighbourhood of $x=0$ ) in the zero viscosity limit is given by

$$
\rho(i \sigma, t) \underset{\sigma \rightarrow 0}{\simeq}-i(2 \pi)^{-1} t_{*}^{-1 / 2}\left(t-t_{*}\right)^{1 / 2} t^{-3 / 2} .
$$

Following reference [19], one expects the discontinuity of the shock to be related to the local density of poles by :

$$
\Delta_{x=0}(t)=u_{\mathrm{L}}\left(0_{-}, t\right)-u_{\mathrm{R}}\left(0_{+}, t\right)=-2 i \pi \rho(0, t) .
$$

Using the odd parity of $u$ and formula (20), one gets

$$
u_{\mathrm{R}}(0, t)=-\frac{1}{2} E^{-3 / 2}\left(t-t_{*}\right)^{1 / 2} t_{*}^{-1 / 2},
$$

which is the correct value obtained directly through equation (8). This pole condensation mechanism thus provides a new interpretation of the known features of the shock (see Fig. 6 and [20]).

\section{Conclusion.}

In this Letter we have analysed the dynamics of Burgers' model for a given paradigmatic initial condition; we found that the preshock and shock are governed by the influence of only two singularities, to which we have associated a topologically simple Riemann surface.

We hope to have made transparent the deterministic case and especially the connection between viscid and inviscid problems. In the statistical problem one expects a much more complicated situation. 


\section{References}

[1] Burgers, J.M., A. K. Verhand-Kon. Nederl. Wetenschappen Afd. Natuurkunde, Eerste Sertie, Vol. 17 (1939) $1-5$.

Burgers, J.M., The Nonlinear Diffusion Equation (D. Reidel, Publ.) 1974.

[2] Germain, P., Méthodes Asymptotiques en Mécanique des Fluides, in Fluid Dynamics (Les Houches Proceed. 1973), R. Balian and J.L. Peube eds. (Gordon and Breach Publ.) 1977.

[3] Fournier, J.D. and Frisch J., J. Mec. Th. Appl. 2 (1983), no 5, 699.

[4] Frisch, U. and Morf, R., Phys. Rev. A 23 (1981) 2673.

[5] Choudnowsky, D.V. and Choodnowsky, G.V., Nuovo Cimento 40B (1977) 339.

[6] Even with complete resummation, certain expansions (e.g. Taylor time series or Wiener-Hermite decomposition (see [7])) yield incorrect scaling laws for the statistical Burgers' velocity field when shocks are present (e.g. $k^{-3}$ for the spatial Fourier energy spectrum, known to behave as $k^{-2}$ ). See a discussion in [3] and references therein.

[7] KaKNG, W.H., Phys. Fluids 13 (1970) 1970.

[8] For small viscosity the " leaders" of the poles (see below and Fig. 6) move near by $x_{\mathrm{s}}$. In both cases their distance to the real axis $\delta(t, v)$ defines the width of the analyticity strip, whose evolution with time may be used as a numerical test (see [9]). In our case it may be obtained explicitly, but formula (9) is generic only for $t \simeq t_{*}$. As explained in reference [10] (see also references therein) this motion may also be obtained with a short-time asymptotic analysis for various equations with initial conditions concentrated in the long wavelength modes (trigonometric polynomials). One of us (J-D-F) acknowledges U. Frisch for discussions on this point. Implications for the statistical mechanics may be found in [4] and [11].

[9] Sulem, C., Sulem, P.L., Frisch, H., J. Comput. Phys. 50 (1983) 138.

[10] Frisch, U., The Analytic Structure of Turbulent Flows, in Chaos and Statistical Mechanics (Kyoto Summer Institute 1983 Proceed.) Y. Kuramoto, ed. (Lecture Notes in Physics, Springer-Verlag) to appear.

[11] Fucito, F., Marchesoni, F., Marinari, E., Parisi, G., Pelmi, L., Ruffo, S., Vulpiani, A., J. Physique 43 (1982) 707.

[12] Hopf, E., Comm. Pure Appl. Mech. 3 (1950) 201 ;

Cole, J.D., Quart. Appl. Math. 9 (1951) 225.

[13] Discussion of linearization and integrability of Burgers' equation in various other senses may be found in [14-17].

[14] Taflin, E., Phys. Rev. Lett. 47 (1981) 1425; erratum 48 (1982) 898.

[15] Degasperis, A. and LeON, J.P., Nuovo Cimento 78B (1983) 129.

[16] Weiss, J., TABor, M., Carnevale, G., J. Math. Phys. 24 (1983) 522. In this paper the Ablowitz-RamaniSegur conjecture is restated and analysed for three P.D.E.'s including Burgers'.

[17] Cornille, H. and Gervois, A., Phys. Lett. 90A (1982) 329.

[18] See e.g. Valiron, G., Théorie des Fonctions, (Masson Publ.) 1942, p. 429.

[19] Lee, T.D. and Yang, C.N., Phys. Rev. 87 (1952) 410.

[20] In particular the known evolution with time of the jump of the shock (Eqs. (21), (22)) grows from zero at $t_{*}$ to a maximum value, while the shock absorbs its neighbourhood, then decays to zero. The dissipation (which does not go to zero in the $v \rightarrow 0_{+}$limit) becoming the dominant effect may be translated in terms of varying density of poles (20). 